\title{
Potential Biochemical Mechanisms of Brain Injury in Diabetes Mellitus
}

\author{
Wei-Xing Ma ${ }^{1,2,3}$, Jing Tang ${ }^{3}$, Zhi-Wen Lei ${ }^{3}$, Chun-Yan $\mathrm{Li}^{1,4}$, Li-Qing Zhao ${ }^{3}$, Chao Lin ${ }^{3}$, Tao \\ Sun $^{3}$, Zheng-Yi Li ${ }^{3}$, Ying-Hui Jiang ${ }^{3}$, Jun-Tao Jia ${ }^{3}$, Cheng-Zhu Liang ${ }^{3}$, Jun-Hong Liu ${ }^{2}$, Liang- \\ Jun Yan $^{1 *}$ \\ ${ }^{1}$ Department of Pharmaceutical, University of North Texas Health Science Center, Fort Worth, Texas, USA \\ ${ }^{2}$ Chemical Engineering Institute, Qingdao University of Science and Technology, Qingdao, Shandong, China \\ ${ }^{3}$ Technological Center, Qingdao Customs, Qingdao, Shandong, China \\ ${ }^{4}$ Shantou University Medical College, Shantou, Guangdong, China
}

[Received July 19, 2019; Revised September 6, 2019; Accepted September 10, 2019]

\begin{abstract}
The goal of this review was to summarize current biochemical mechanisms of and risk factors for diabetic brain injury. We mainly summarized mechanisms published in the past three years and focused on diabetes induced cognitive impairment, diabetes-linked Alzheimer's disease, and diabetic stroke. We think there is a need to conduct further studies with increased sample sizes and prolonged period of follow-ups to clarify the effect of DM on brain dysfunction. Additionally, we also think that enhancing experimental reproducibility using animal models in conjunction with application of advanced devices should be considered when new experiments are designed. It is expected that further investigation of the underlying mechanisms of diabetic cognitive impairment will provide novel insights into therapeutic approaches for ameliorating diabetes-associated injury in the brain.
\end{abstract}

Key words: diabetes, brain, cognitive impairment, Alzheimer's disease, stroke

\section{Introduction}

Diabetes mellitus (DM), including Type 1 and Type 2 $\mathrm{DM}$, is a chronic disease that can damage many organs in the body, leading to symptoms such as hypertension, vision loss, cerebrovascular disease, cerebral edema and infarction. DM can also induce cortical and subcortical atrophy, white matter abnormalities, and perturbation of glymphatic function as well as overall dysregulation of cerebral metabolism [1-6]. Indeed, it has been established that DM-induced central nervous system (CNS) complications can cause cerebrovascular change and hippocampal synaptic plasticity alterations thereby contributing to reduced impulse condition velocity in presynaptic fibers and brain atrophy [7-9]. Additionally, several studies have also demonstrated that DM can induce $\mathrm{Ca}^{2+}$ dysregulation and accelerate brain aging that is closely related to $\mathrm{Ca}^{2+}$ dyshomeostasis [10]. Notably, it has been suggested that even if appropriate glucose control can limit the development of these severe complications, such limitation may not be sufficient to entirely prevent these complications from occurring [8].

The brain, as one of the most sophisticated organ in our body [11], plays an important role in glucose homeostasis by producing adaptive changes in energy intake, energy consumption, and hepatic glucose

*Correspondence should be addressed to: Dr. Liang-Jun Yan, Department of Pharmaceutical, University of North Texas Health Science Center, Fort Worth, Texas, USA. Email: liang-jun.yan@unthsc.edu.

Copyright: () 2019 Ma WX et al. This is an open-access article distributed under the terms of the Creative Commons Attribution License, which permits unrestricted use, distribution, and reproduction in any medium, provided the original author and source are cre dited. 
production [12]. Many cerebral-related injuries, disorders or dysfunctions can lead to irreparably serious consequences. Accumulating evidence indicates that more and more cerebral dysfunction is likely to be attributed to DM-related complications [7, 13-20], which might directly contribute to psychological and mental health outcomes [21] as well as other neurological deficits [22].

While stable glucose level is essential for cerebral metabolism and neuronal function and activity, prolonged periods of low or high glucose levels as well as rapid glucose fluctuations can result in neuronal injury [13]. Therefore, elucidating the mechanism of diabetic brain injury has been an increasing research interest. In this review, we attempted to summarize the current major biochemical mechanisms of diabetic brain injury, including hyperglycemia-induced cognitive impairment, diabetic Alzheimer's disease (AD) and diabetic stroke. It should be noted that our review is not meant to exhaust all the possible mechanisms of diabetic brain injury documented in the literature.

\section{Potential Mechanism in the Development of Diabetic Cognitive Impairment}

\subsection{Diabetes enhanced cerebral lesion deteriorates cognitive impairment}

Increasing evidence indicated that $\mathrm{DM}$ is related to cognitive impairment, and the level of hyperglycemia and duration of DM have been shown to be linked with cognitive decline [23]. Cerebrovascular dysfunction is associated with cognitive impairment in patients with vascular dementia [24]. Abnormal variations in acetylcholine production, transport mechanism of choline, degradation of nicotinic and muscarinic receptors in the cerebral cortex can all result in cognitive impairment [25]. Recent analysis has revealed that gray matter atrophy and lesion of white material were related to declined cognitive performance that can be attributed to diabetes [14]. Cognition is particularly impaired when diabetes onset occurs during the midlife stage. Mid-aged adults with type 2 diabetes have exhibited alterations in neural function. In particular, reduced neural activation has been observed by functional imaging studies, indicating that cerebral changes probably precede diabetes onset in high risk individuals [26]. Another study based on advanced multimodal MRI showed that patients with impairment had a lower gray matter volume especially in the right temporal lobe and subcortical brain region. Concurrently, white matter volume and connectivity were decreased when compared to those without cognitive impairment [16]. Emerging evidence revealed by proton magnetic resonance spectroscopy also shows that adults with DM had a 9.3\% lower ratio of $\mathrm{N}$ acetylaspartate/creatine (NAA/cre) than in healthy subjects who were without DM. Thus, decreased NAA could be a potential biomarker of central neuronal dysfunction and may be useful in evaluating progression of neuropathy in DM [17]. Moreover, studies focusing on brain derived neurotrophic factor (BDNF), one of the most essential neurotrophic factors in the brain [27], and dipeptidyl peptidase-4 (DPP4), initially identified as a therapeutic target for type 2 diabetes [28], have demonstrated that both BDNF and DPP4 function as an antigenic enzyme involved in hyperglycemia, oxidative stress and inflammation-associated insulin resistance [29]). These studies provide evidence for the role of increased DPP4 and decreased BDNF in type 2-DMrelated cognitive dysfunction [30], suggesting that DPP4 to BDNF ratio could be a novel biomarker for DM-related cognitive impairment [31].

\subsection{Diabetes enhanced $\mathrm{ca}^{2+}$ dysregulation is a vital pathogenic mechanism of cognitive impairment}

Tight regulation of calcium signaling is critical for many important functions of the brain, and subtle impairment in calcium signaling or disturbed calcium homeostasis can result in deleterious consequences including neuronal death [32] and age-related cognitive decline [33-35]. DM increases the risk of developing renal failure and contributes to impediment to phosphorus excretion from kidney, and thus leads to hyperphosphatemia. Under hyperphosphatemic condition, hypocalcemia is induced by interfering with phosphorus excretion. Moreover, phosphate can bind ionized calcium and remove calcium from the bloodstream [36]. As $\mathrm{Ca}^{2+}$ is indispensable for insulin and glucagon secretion, alterations in $\mathrm{Ca}^{2+}$ homeostasis caused by DM can thus impact blood glucose [37]. Many studies suggest that $\mathrm{Ca}^{2+}$ dysregulation could be a pivotal target of DM and might mediate the deleterious effects of DM on hippocampal function and behavior [10, 38]. Moreover, accumulation of intracellular free $\mathrm{Ca}^{2+}$ could also decrease the levels of $\mathrm{Ca}^{2+} /$ calmodulin-dependent protein kinase II (CaMKII). As CaMKII regulated cAMP-response element binding protein (CREB) is involved in maintaining appropriate cognitive formation, disruption of calcium signaling by diabetic hyperglycemia can thus result in cognitive impairment [38] (Figure 1). Conversely, modulation of calcium-binding protein apoaequorin could have positive effects in treating cognitive dysfunction [39, 40]. Additionally, the transport of calcium across the inner mitochondrial membrane could also play a key role in neuronal physiology and pathology [41]. It has been reported that change in calcium signaling is likely involved in the compromise of mitochondrial ATP 
production [42] implicated in neurodegeneration [43]. $\mathrm{Ca}^{2+}$ signals in mitochondria stimulate ATP synthesis which is an important part of excitation-metabolic coupling. The communication between mitochondria and endoplasmic reticulum also plays an essential role in this metabolic fine tuning. And this communication can be impaired by DM leading to reduced cognitive functions [44]. Moreover, it has been demonstrated that a close relationship exists between mitochondrial dysfunction and cellular calcium homeostasis, which is likely contributed by metabolic increase in cytosolic free calcium and variations in intracellular calcium dynamics [45].

\subsection{Diabetes enhanced blood-brain barrier (BBB)} breakdown is possibly related to cognitive impairment

BBB forms a pivotal interface between the CNS and the general circulation [46] and contributes to CNS homeostasis [47]. Although mechanism underlying BBB dysfunction is missing [48], recent evidence indicates that BBB breakdown is prevalent, functionally relevant, and closely related to more rapid cognitive impairment [49]. Chronically increased oxidative stress, as one of the common pathological characteristics in DM, directly results in dysregulation of BBB tight junction proteins. This alteration, concurrent with DM hyperglycemia, may also increase BBB permeability [50] and result in further oxidative damage and glucose leakage from the blood to the brain parenchyma [51, 52]. DM-associated brain microvascular damage can also compromise BBB function, leading to cognitive impairment detected in neurodegenerative disorders [53]. Adaptive homeostatic responses at $\mathrm{BBB}$ might cope with increased oxygen and nutrient demand by regulation of influx and efflux via BBB transporters, which could change microvessel permeability, disrupts cerebral microenvironment and eventually leads to cognitive impairment [54]. Additionally, DM can trigger an increase in production of reactive oxygen species (ROS) and reactive nitrogen species (RNS) and a decrease in antioxidant enzyme activities, thereby accentuates brain damage [55] (Figure $1)$.

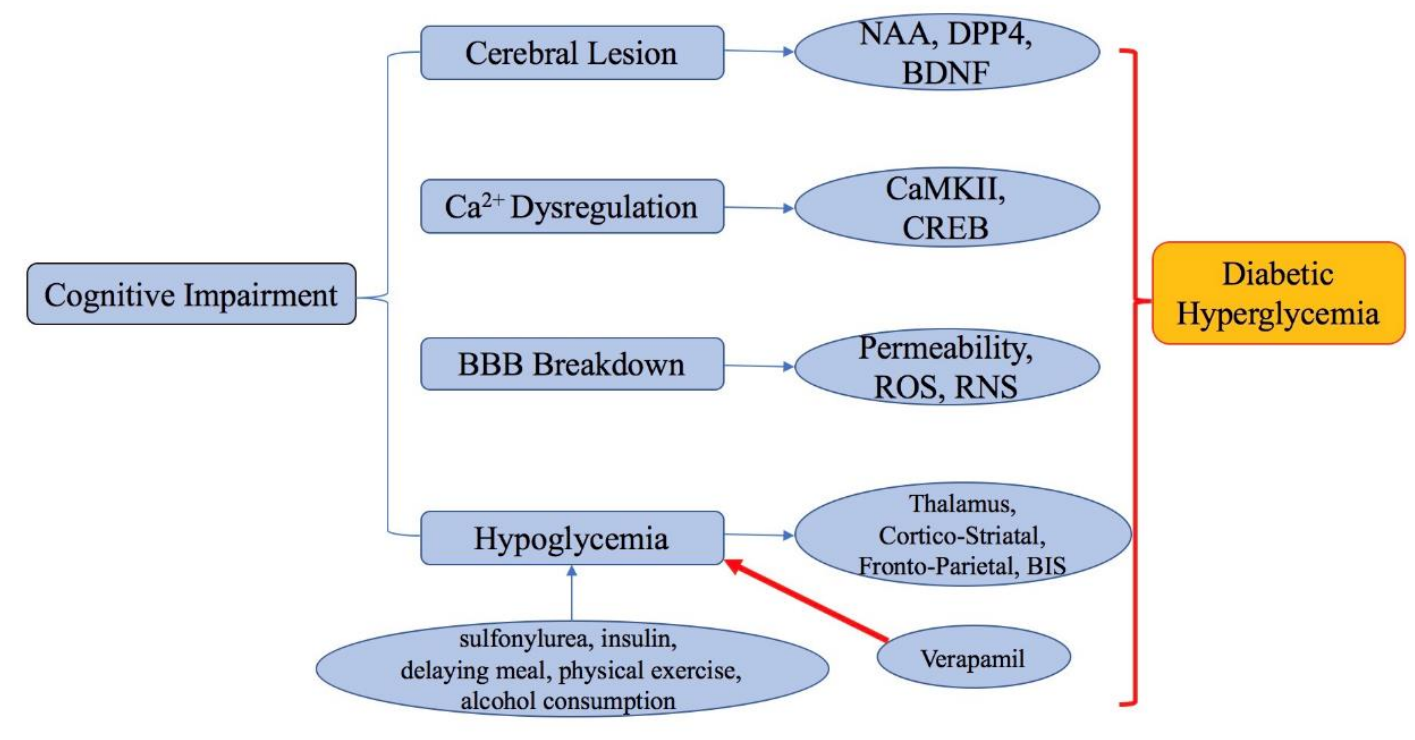

Figure 1. Signaling pathways and risk factors that are potentially involved in development of cognitive impairment induced by diabetes.

Over the past few decades, plenty of proteins belonging to the series of angioneurins have been considered as potential therapeutic targets for CNS caused by BBB dysfunction. A more recent review article states that certain angioneurins, including vascular endothelial growth factors, angiopoietins, platelet-derived growth factors and erythropoietin, are critical regulators of $\mathrm{BBB}$ integrity during brain injury and may vitally determine disease progression by multifaceted effects on the compromised BBB. These effects are based on the functional variety of angioneurins, which includes enhancing neuronal survival, stimulating the recruitment and proliferation of neural precursor cells, promoting neuronal plasticity, maintaining vascular integrity, and 
improving BBB tightness [56]. It has also been reported that activated brain mast cell, as the "first responder" in brain injury, may also contribute to CNS inflammation and cognitive dysfunction by promoting BBB disruption [57].

\subsection{Diabetic hypoglycemia leads to cognitive impairment}

Diabetic patients are exposed frequently to either hyperglycemia or hypoglycemia [58]. Hyperglycemia and hypoglycemia are two sides of a same coin. Hypoglycemia is a common consequence of diabetes treatment. There are several factors that could lead to hypoglycemia, including sulfonylurea therapy, insulin therapy, delaying or missing a meal, less physical activity, or increased alcohol consumption [59]. Exposure to hypoglycemia can cause a redistribution of cerebral blood flow towards the thalamus [60], and lead to brain damage and cognitive impairment [61-63]. Acute hypoglycemia decreases cerebral glucose consumption and might result in permanent brain injury. The areas of hypoglycemiaactivated decrease in glucose consumption is more prominent in those of the brain where normal cerebral glucose consumption is higher [64]. However, hypoglycemia cannot be detected during general anesthesia, except for intermittent blood glucose monitoring or electroencephalogram readings. For diabetic patients, when the intraoperative bispectral index (BIS) value is abnormally low, hypoglycemia should be considered $[64,65]$. One recent research concludes that, in patients (especially Type $1 \mathrm{DM}$ ) with hypoglycemia unawareness, there is a developing blunting of cerebral responses in cortico-striatal and fronto-parietal neurocircuits in response to mild-moderate hypoglycemia. That's why certain patients fail to respond to falling blood glucose levels [66]. In addition, another recent research states that calcium channel blocker, verapamil, could significantly decrease cerebral damage and cognitive impairment caused by severe hypoglycemia, which may be a useful approach to prevent hypoglycemia-induced cerebral injury [67]. It should be noted that while chronic hypoglycemia causes increased transportation, utilization and preservation of glucose in the brain, hyperglycemia-induced aggravation of ischemic brain injury is thought to be caused by increase in glucose concentration in the brain [68].

\section{Potential DM-induced mechanisms leading to AD}

$\mathrm{AD}$, thought to be type $3 \mathrm{DM}[69,70]$, is the most frequent form of dementia and a growing public health burden. AD results in aggregation of amyloid $\beta(\mathrm{A} \beta)$ peptides in the brain, dysregulation of tau protein phosphorylation, insulin-resistance, lipid dysregulation, axonal loss, memory loss, and neuronal death [71-74]. The aforementioned $\mathrm{BBB}$ damage also could promote $\mathrm{AD}$ development [75]. It is well-established that DM increases the risk of $\mathrm{AD}$ and may play a causative role in the progress of $\mathrm{AD}$ pathogenesis [76].

Relevant studies suggest that the potential mechanisms of diabetes-induced $\mathrm{AD}$ might be due to dysregulation of glucose and insulin signaling in Type 2 DM. It is thought that insulin deficiency due to $\beta$ cell dysfunction in full-blown Type $2 \mathrm{DM}$ may cause AD by hampering insulin's function in the brain [77-79]. Insulin presents critical functions in the CNS that goes far beyond glucose metabolism [80]. It is currently believed that aberrancy of glycogen synthase kinase-3 (GSK-3) could lead to $\mathrm{AD}$ [81], and GSK-3 $\beta$ might be the potential bridge between DM and AD. This is because GSK-3 $\beta$ abnormality could result in insulin resistance and insulin deficiency, thereby leading to phosphorylation dysregulation of tau protein (a pathological feature in $\mathrm{AD}$ ) $[82,83]$.

It is also thought that inflammatory mechanisms can accelerate the core $\mathrm{AD}$ pathology development [84]. It has been reported that inflammation disrupts normal cerebral insulin signaling [85] and is caused by the accumulation of $A \beta$ in the brain. Therefore, inflammation is considered a causative point in $\mathrm{AD}$ and is also thought to be an underlying link between $\mathrm{AD}$ and Type $2 \mathrm{DM}$ [86]. Another factor receiving a lot of attention is toll-like receptors (TLRs), especially toll-like receptor 4 (TLR4). A $\beta$-mediated TLR4 activation deteriorates neuroinflammation in AD [87]. Chronic TLR4 activation may result in insulin resistance in $\mathrm{DM}$ and $\mathrm{A} \beta$ deposition in $\mathrm{AD}$, and may serve as a potential link between DM and AD [88]. Moreover, GSK-3 pathway, dysregulated by insulin resistance, enhances the $A \beta$ plaque pathology and is closely related to tau misfolding and toxicity [89]. It should be noted that accumulation of $A \beta$ modified by DM has been observed in wild type animals and animal models of $\mathrm{AD}$, but not in humans [90]. The third important factor is nuclear factor- $\kappa \mathrm{B}(\mathrm{NF} \kappa \mathrm{B})$. Hypothalamic inflammation activates the proinflammatory $\mathrm{NF} \kappa \mathrm{B}$ pathway, leading to glucose intolerance and insulin resistance. Moreover, within CNS, NFkB-driven hypothalamic inflammation employs a mechanism to induce the loss of blood pressure homeostasis, which is likely in parallel to producing glucose intolerance and insulin resistance [91]. TLRs aforementioned are primarily conveyed by the activation of NFKB which is a key early event in the pathobiology of $\mathrm{DM}$ and its complications. As a master regulator of brain inflammation, NFkB controls the expression of hundreds of genes implicated in innate immune responses, induces cytotoxicity, promotes cell division and apoptosis, and has a strategic role in the link between oxidative stress and inflammation [92]. Additionally, a recent research 
demonstrates that Type $2 \mathrm{DM}$ with diabetic ketoacidosis (DKA) increases risk of AD [93]. Another recent research indicates that there is a correlation between apolipoprotein E polymorphism and the risk of $\mathrm{AD}$, and $\varepsilon 3 / 4$ and $\varepsilon 4$ genotype of ApoE are the pathogenic factor for $\mathrm{AD}$ in patients with Type 2 DM [94]. Moreover, in terms of lipid regulation, ApoE is essential for lipid homeostasis, lipoprotein catabolism and cholesterol transport into cells. Under DM condition, there is an increased cholesterol flux in patients with $\mathrm{AD}$ [73]. It should also be pointed out that advanced glycation end-products (AGEs) are also involved in the complications of DM and are critical to the pathogenesis of AD. Patients suffering from AD combined with DM present an increase in cell damage via a receptor-for-AGEs-dependent mechanism, indicating that AGEs may enhance the vicious cycle of oxidative stress [95].

Therefore, it is clear that there are common pathophysiological mechanisms between DM and AD. Role of insulin and insulin resistance are the key nexus. When insulin receptor activity is hampered by DM, hypoglycemia could be induced which constrains glucose metabolism in the brain. That's why diabetic patients are more at risk of deteriorating $\mathrm{AD}$, and $\mathrm{AD}$ is called "Type 3 DM" $[96,97]$. It should be pointed out that mechanisms underlying the alterations in macrovascular and microvascular cerebral blood flow (CBF) induced by DM should be further explored, because reduced CBF may initiate a series of events promoting cognitive impairment in $\mathrm{AD}[98,99]$.

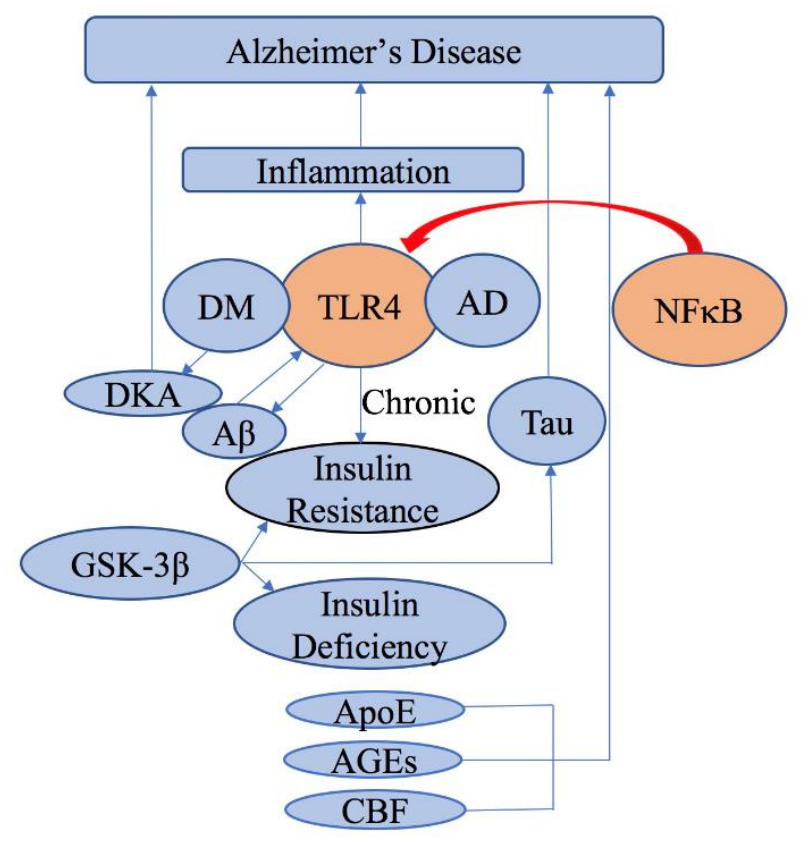

Figure 2. Signaling pathways and risk factors that are potentially involved in diabetic AD.
While many relevant studies have been conducted, the potential mechanisms linking $\mathrm{DM}$ to $\mathrm{AD}$ are still under debate [100]. We think it is necessary to carry out further studies with increased sample sizes and extended period of follow-ups to elucidate the effect of DM on cerebral damage in patients with $\mathrm{AD}$ [97]. Fig. 2 depicts all the potential mechanisms of diabetes-induced $\mathrm{AD}$ as described above.

\section{Potential DM-induced mechanisms leading to Diabetic Stroke}

$\mathrm{DM}$ is an increased risk factor for stroke; and about one fifth of patients with DM could die from stroke. In particular, DM duration has been shown to increase the risk of ischemic stroke (IS); with each additional year of DM duration, the risk for stroke is increased by 3\% [101]. Stroke, especially IS, is currently the second leading cause of death and second cause of dementia after AD and often arises together with Type 2 DM [102, 103]. Mechanisms underlying DM may worsen consequence including vascular damage with stenosis of cerebral arteries leading to impairment of cerebral collateral flow [104]. Patients with DM presented an increased recurrence of stroke, especially IS, leading to neuronal cell death and infarction that accounts for about $87 \%$ of all strokes [63, 105, 106] when compared to non-DM patients [107, 108]. Patients suffering from acute ischemic stroke (AIS) with a history of prior stroke and DM could have increased serious cerebral injury [109]. A recent study focusing on the impact of DM on AIS also demonstrates that stroke recurrence was dramatically higher in DM patients than in prediabetic patients and healthy subjects $(4.2 \%$ versus 0.7 versus 2.2\%; $\mathrm{P}=0.005$ ) [110]. And prediabetes, upon meta-analysis including big population, was also shown to be involved in increasing risk of new stroke in comparison to patients with IS or transient ischemic attack stroke (TIA) [111].

Figure 3 presents the general pathways and risk factors involved in diabetic stroke discussed here. With DM being a comorbidity, patients with IS, TIA and intracerebral hemorrhage (ICH) had higher mortality [112]. The neurovascular unit (NVU) can be constrained by stroke, or hyperglycemia combined with DM, which serves as a common comorbidity in patients with AIS. Maintaining the NVU integrity and protecting NVU against damage are approaches to treating AIS. However, undesirable consequence such as AIS conversion to a worse hemorrhagic stroke (HS) is possible [113]. Thus, treatments for lowering glucose and decreasing vascular risk factors for stroke prevention should be urgently investigated [102]. Severity of loss of pericytes after an ischemic event may have important implications for stroke recovery but direct evidence is lacking. However, 
in terms of the crucial determent of pericyte density in vascular reparative response, it can only be speculated at this moment that extensive loss of pericytes (or pericytes injury) contributes to stroke and post-stroke cerebral vasoregression in diabetes. This is so as the relationship between pericyte loss or injury and stroke injury remains to be investigated. [18, 114]. It should be pointed out that post-stroke depression/anxiety is also a common neuropsychological consequence of IS [115]. In addition, brain edema is the primary cause of IS mortality. The mechanism underlying this phenomenon may be the rapid disturbance of water contributing to abnormal elevation in intracranial pressure and/or intracranial fluid $[116,117]$.

Therefore, while antidiabetic medicines for stroke in patients with DM are vigrously being developed [118], further studies should also concentrate on potential mechanism of DM-induced different phase of stroke [119]. This might be helpful in identifying specific pathogenic mechanisms underlying cerebral damage caused by DM potentiated stroke. However, most of animal model studies focusing on stroke research need animal sacrifice, which limits the ensuing cognition or behavioral studies on the same animal. Additionally, reproducibility may also be an issue. It seems that the novel approach using magnetic resonance imaging for stroke evaluation may enhance reproducibility [120].

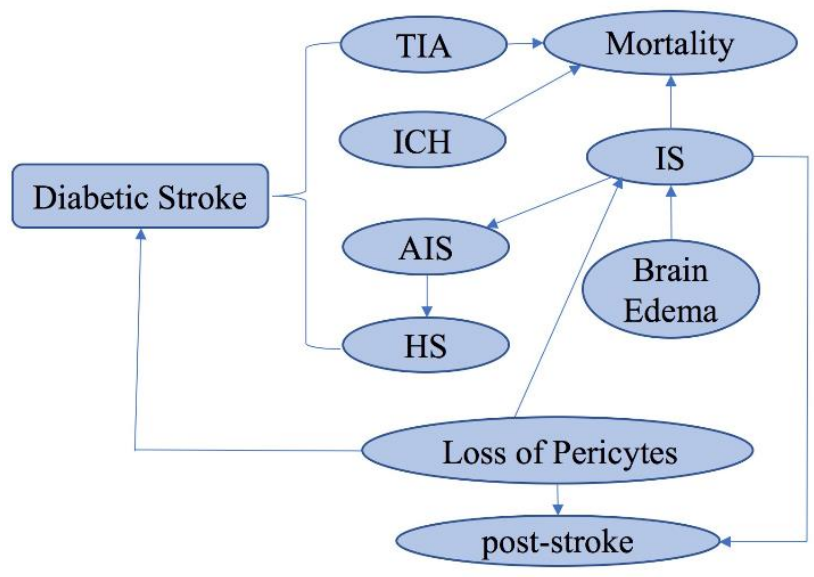

Figure 3. Signaling pathways and risk factors potentially involved in diabetic stroke.

\section{Summary}

In this review, we have summarized current biochemical mechanisms of diabetic brain injury based on publications published mainly between 2017 and 2019, which accounts for more than two-thirds of all the citations. While many published articles elucidated similar mechanisms in different perspectives, conditions, experiments, hypotheses and explanations, certain controversies remained common among these articles. Further investigations combining better-designed animal models with advanced and high-precision devices are badly needed to enhance reproducibility of experiments conducted by disparate investigators.

\section{Conflict of Interest}

The authors declare that there is no conflict of interest regarding the publication of this article.

\section{References}

[1] Roriz-Filho JS, Sá-Roriz TM, Rosset I, Camozzato AL, Santos AC, Chaves MrLF, et al. (2009). (Pre) diabetes, brain aging, and cognition. Biochim Biophys Acta, 1792:432-443.

[2] Toth C (2014). Chapter 32-Diabetes and neurodegeneration in the brain. Handbook of Clinical Neurology, 126:489-511.

[3] Guo S, Deng W, Xing C, Zhou Y, Ning M, Lo EH (2019). Effects of aging, hypertension and diabetes on the mouse brain and heart vasculomes. Neurobiol Dis, 126:117-123.

[4] Eisenhut M (2018). In diabetic ketoacidosis brain injury including cerebral oedema and infarction is caused by interleukin-1. Med Hypotheses, 121:44-46.

[5] Vargas R, Rincón J, Pedreañez A, Viera N, HernándezFonseca JP, Peña C, et al. (2012). Role of angiotensin II in the brain inflammatory events during experimental diabetes in rats. Brain Res, 1453:64-76.

[6] Wisse LEM, Bresser Jd, Geerlings MI, Reijmer YD, Portegies MLP, Brundel M, et al. (2014). Global brain atrophy but not hippocampal atrophy is related to type 2 diabetes. J Neurol Sci, 344:32-36.

[7] Wrighten SA, Piroli GG, Grillo CA, Reagan LP (2009). A look inside the diabetic brain: Contributors to diabetesinduced brain aging. Biochim Biophys Acta, 2009:444453.

[8] Biessels GJ, Heide LPvd, Kamal A, Bleys RLAW, Gispen WH (2002). Ageing and diabetes: implications for brain function. Eur J Pharmacol, 441:1-14.

[9] Chen Z, Li L, Sun J, Ma L (2012). Mapping the brain in type II diabetes: Voxel-based morphometry using DARTEL. Eur J Radiol, 81:1870-1876.

[10] Thibault O, Anderson KL, DeMoll C, Brewer LD, Landfield PW, Porter NM (2013). Hippocampal calcium dysregulation at the nexus of diabetes and brain aging. Eur J Pharmacol, 719:34-43.

[11] Schoenemann PT (2006). Evolution of the Size and Functional Areas of the Human Brain. Annu Rev Anthropol, 35:379-406.

[12] Rosenberg J, Lechea N, Pentang GN, Shah NJ (2019). What magnetic resonance imaging reveals - A systematic review of the relationship between type II diabetes and associated brain distortions of structure and cognitive functioning. Front Neuroendocrinol, 52:79112. 
[13] Cameron FJ (2015). The Impact of Diabetes on Brain Function in Childhood and Adolescence. Pediatr Clin N Am, 62:911-927.

[14] Hughes TM, Sink KM, Williamson JD, Hugenschmidt CE, Wagner BC, Whitlow CT, et al. (2018). Relationships between cerebral structure and cognitive function in African Americans with type 2 diabetes. Journal of Diabetes and Its Complications, 32:916-921.

[15] Bancks MP, Alonso A, Gottesman RF, Mosley TH (2017). Brain function and structure and risk for incident diabetes: The Atherosclerosis Risk in Communities Study. Alzheimer's \& Dementia: the Journal of the Alzheimer's Association, 13:1345-1354.

[16] Groeneveld O, Reijmer Y, Heinen R, Kuijf H, Koekkoek P, Janssen J, et al. (2018). Brain imaging correlates of mild cognitive impairment and early dementia in patients with type 2 diabetes mellitus. Nutrition, Metabolism \& Cardiovascular Diseases, 28:1253-1260.

[17] Hansen TM, Brock B, Juhl A, Drewes AM, Vorum H, Andersen CU, et al. (2019). Brain spectroscopy reveals that $\mathrm{N}$-acetylaspartate is associated to peripheral sensorimotor neuropathy in type 1 diabetes. Journal of Diabetes and Its Complications, 33:323-328.

[18] Ergula A, Valenzuela JP, Fouda AY, Fagan SC (2015). Cellular connections, microenvironment and brain angiogenesis in diabetes: Lost communication signals in the post-stroke period. Brain Res, 1623:81-96.

[19] Arnal E, Miranda M, Johnsen-Soriano S, J.Romero F (2014). The Anti-Oxidative Component of Docosahexaenoic Acid (DHA) in the Brain in Diabetes. Diabetes: Oxidative Stress and Dietary Antioxidants, Chapter 13:129-134.

[20] Kooistra M, Geerlings MI, Mali WPTM, Vincken KL, Graaf Yvd, Biessels GJ (2013). Diabetes mellitus and progression of vascular brain lesions and brain atrophy in patients with symptomatic atherosclerotic disease. The SMART-MR study. J Neurol Sci, 332:69-74.

[21] MD FJC, Northam EA, Ryan CM (2019). The effect of type 1 diabetes on the developing brain. The Lancet Child \& Adolescent Health, 3:427-436.

[22] Shahid MH, Verma A, Youngblood L (2018). From Mechanical to Chemical: A Case of Diabetes Insipidus Induced by Concussive Brain Injury. Am J Med, 131:e293-e294.

[23] Wu Y, Ye L, Yuan Y, Jiang T, Guo X, Wang Z, et al. (2019). Autophagy Activation is Associated with Neuroprotection in Diabetes-associated Cognitive Decline. Aging Dis, 10:1-13

[24] Park SY, Kim HY, Lee YS, Heo HJ, Shin HK, Lee WS, et al. (2019). Augmented improvement of cognition and memory by aripiprazole add-on for cilostazol treatment in the chronic cerebral hypoperfusion mouse model. Behav Brain Res, 365:133-140.

[25] M.A. A, Kumar MKM, Sabitha M, Menon KK, Nair SC (2019). Nanotechnology approaches for enhanced CNS delivery in treating T Alzheimer's disease. J Drug Deliv Sci Tec, 51:297-309.

[26] Macpherson H, Formica M, Harris E, Daly RM (2017). Brain functional alterations in Type 2 Diabetes - A systematic review of fMRI studies. Front Neuroendocrinol, 47:34-46.

[27] Song H-D, Kim SN, Saha A, Ahn S-Y, Akindehin S, Son $\mathrm{Y}$, et al. (2020). Aging-Induced Brain-Derived Neurotrophic Factor in Adipocyte Progenitors Contributes to Adipose Tissue Dysfunction. Aging Dis, 11:1-13.

[28] Yang L, Yuan J, Zhou Z (2014). Emerging Roles of Dipeptidyl Peptidase 4 Inhibitors: Anti-Inflammatory and Immunomodulatory Effect and Its Application in Diabetes Mellitus. Can J Diabetes, 38:473-479.

[29] Qiao J, Li L, Ma Y, Shi R, Teng M (2018). Biological function of dipeptidyl peptidase-4 on type 2 diabetes patients and diabetic mice. Curr Res Translational Med, 67:89-92.

[30] Abdelwaheda OM, Tork OM, Din MMGe, L. Rashedb MZ (2018). Effect of glucagon-like peptide-1 analogue; Exendin-4, on cognitive $T$ functions in type 2 diabetes mellitus; possible modulation of brain derived neurotrophic factor and brain Visfatin. Brain Res Bull, 139:67-80.

[31] Zheng T, Liu H, Qin L, Chen B, Zhang X, Hu X, et al. (2018). Oxidative stress-mediated influence of plasma DPP4 activity to BDNF ratio on mild cognitive impairment in elderly type 2 diabetic patients: results from the GDMD study in China. Metab, 87:105-112.

[32] Chandran R, Kumar M, Kesavan L, Jacob RS, Gunasekaran S, Lakshmi S, et al. (2019). Cellular calcium signaling in the aging brain. J Chem Neuroanat, 95:95-114.

[33] Navakkode S, Liu C, Soong TW (2018). Altered function of neuronal L-type calcium channels in ageing and neuroinflammation:Implications in age-related synaptic dysfunction and cognitive decline. Aging Res Rev, 42:86-99.

[34] Grützner TM, Listunova L, Fabian GA, Kramer BA, Flach D, Weisbrod M, et al. (2018). Serum calcium levels and neuropsychological performance in depression and matched healthy controls: Reversal of correlation a marker of the aging cognitive clock? Psychoneuroendocrino, 91:198-205.

[35] Sharma A, Schray A, Bartolovic M, Roesch-Ely D, Aschenbrenner S, Weisbrod M (2017). Relationship between serum calcium and neuropsychological performance might indicate etiological heterogeneity underlying cognitive deficits in schizophrenia and depression. Psychiatry Res, 252:80-86.

[36] Ahn C, Kang J-H, Jeung E-B (2017). Calcium homeostasis in diabetes mellitus. J Vet Sci, 18:261-266.

[37] Arruda AP, S.Hotamisligil G (2015). Calcium Homeostasis and Organelle Function in the Pathogenesis of Obesity and Diabetes. Cell Metab, 22:381-397.

[38] Ouyang L, Zhang W, Du G, Liu H, Xie J, Gu J, et al. (2019). Lead Exposure-Induced Cognitive Impairment through RyR-Modulating Intracellular Calcium Signaling in Aged Rats. Toxicology, 419:55-64.

[39] Underwood M, Sivesind P, Gabourie T (2011). Effect of apoaequorin on cognitive function. Alzheimer's \& Dementia, 7:e65.

[40] Milgram NW, Landsberg G, Merrick D, Underwood MY 
(2015). A novel mechanism for cognitive enhancement in aged dogs with the use of a calcium-buffering protein. J Vet Behav, 10:217-222.

[41] Nicholls DG (2017). Brain mitochondrial calcium transport: Origins of the set-point concept and its application to physiology and pathology. Neurochem Int, 109:5-12.

[42] Workgroup AsACH (2017). Calcium Hypothesis of Alzheimer's disease and brain aging: A framework for integrating new evidence into a comprehensive theory of pathogenesis. Alzheimer's \& Dementia, 13:178-182.

[43] Rossi A, Pizzo P, Filadi R (2019). Calcium, mitochondria and cell metabolism: A functional triangle in bioenergetics. BBA-Mol Cell Res, 1866:1068-1078.

[44] Guerrero-Hernandez A, Verkhratsky A (2014). Calcium signalling in diabetes. Cell Calcium, 56:297-301.

[45] Jaworska A, Dzbek J, Styczynska M, Kuznicki J (2013). Analysis of calcium homeostasis in fresh lymphocytes from patients with sporadic Alzheimer's disease or mild cognitive impairment. BBA-Mol Cell Res, 1833:16921699.

[46] M.Gray S, J.Barrett E (2018). Chapter 11 - The BloodBrain Barrier in Diabetes Mellitus. Type 2 Diabetes and Dementia:211-229.

[47] Jiang X, Andjelkovic AV, Zhu L, Yang T, Bennett MVL, Chen J, et al. (2018). Blood-brain barrier dysfunction and recovery after ischemic stroke. Prog Neurobiol, 163164:144-171.

[48] Gelb S, Stock AD, Anzi S, Putterman C, Ben-Zvi A (2018). Mechanisms of neuropsychiatric lupus: The relative roles of the blood-cerebrospinal fluid barrier versus blood-brain barrier. J Autoimmun, 91:34-44.

[49] Bowman GL, Dayon L, Kirkland R, Wojcik J, Peyratout G, Severin IC, et al. (2018). Blood-brain barrier breakdown, neuroinflammation, and cognitive decline in older adults. Alzheimer's \& Dementia, 14:1640-1650.

[50] Duarte-Delgado NP, Vásquez G, Ortiz-Reyes BL (2019). Blood-brain barrier disruption and neuroinflammation as pathophysiological mechanisms of the diffuse manifestations of neuropsychiatric systemic lupus erythematosus. Autoimmun Rev, 18:426-432.

[51] Brook E, Mamo J, Wong R, Al-Salami H, Falasca M, Lam V, et al. (2019). Blood-brain barrier disturbances in diabetes-associated dementia: Therapeutic potential for cannabinoids. Pharmacol Res, 141:291-297.

[52] Xu Z, WenZeng, Sun J, Chen W, Zhang R, Yang Z, et al. (2017). The quantification of blood-brain barrier disruption using dynamic contrast-enhanced magnetic resonance imaging in aging rhesus monkeys with spontaneous type 2 diabetes mellitus. NeuroImage, 158:480-487.

[53] Janelidze S, Hertze J, Nägga K, Nilsson K, Nilsson C, Group tSBS, et al. (2017). Increased blood-brain barrier permeability is associated with dementia and diabetes but not amyloid pathology or APOE genotype. Neurobiol Aging, 51:104-112.

[54] C.Lim D, I.Packa A (2014). Obstructive sleep apnea and cognitive impairment: Addressing the blood-brain barrier. Sleep Med Rev, 18:35-48.

[55] Mohamed NA, Gawad HSA (2017). Taurine dietary supplementation attenuates brain, thyroid, testicular disturbances and oxidative stress in streptozotocininduced diabetes mellitus in male rats. Beni-Suef University Journal of Basic and Applied Sciences, $6: 247-252$

[56] Kunze R, H.Marti H (2019). Angioneurins - Key regulators of blood-brain barrier integrity during hypoxic and ischemic brain injury. Prog Neurobiol, 178:1-29.

[57] Zhang S, Dong H, Zhang X, Li N, Sun J, Qian Y (2016). Cerebral mast cells contribute to postoperative cognitive dysfunction by promoting blood brain barrier disruption. Behav Brain Res, 298:158-166.

[58] Patell R, Nigmatoulline D, Bena J, Kim DG, MessingerRapport B, Lansang MC (2017). Hyperglycemia and Hypoglycemia in Patients with Diabetes in Skilled Nursing Facilities. Endocr Pr, 23:458-465.

[59] Tourkmani AM, Alharbi TJ, Rsheed AMB, AlRasheed AN, AlBattal SM, Abdelhay O, et al. (2018). Hypoglycemia in Type 2 Diabetes Mellitus patients: A review article. Diabetes \& Metabolic Syndrome: Clin Res Rev, 12:791-794.

[60] Wiegers EC, Becker KM, Rooijackers HM, SamsonHimmelstjerna FCv, Tack CJ, Heerschap A, et al. (2016). Cerebral blood flow response to hypoglycemia is altered in patients with type 1 diabetes and impaired awareness of hypoglycemia. J Cereb Blood Flow Metab, 37:19942001.

[61] Sheen Y-J, Sheu WHH (2016). Association between hypoglycemia and dementia in patients with type 2 diabetes. Diabetes Res Clin Pr, 116:279-287.

[62] Yuan L, Lingling Z, Ling C, Liting C, Hao L, Huiping L, et al. (2017). Correlation between blood glucose fluctuation and brain damage in neonates with hypoglycemia. J Clin Pediatr, 35:652-654.

[63] Shukla V, Shakya AK, Perez-Pinzon MA, Dave KR (2017). Cerebral ischemic damage in diabetes: an inflammatory perspective. J Neuroinflammation, 14:122.

[64] Rehni AK, Dave KR (2018). Impact of Hypoglycemia on Brain Metabolism During Diabetes. Mol Neurobiol, 55:9075-9088.

[65] Xi C, Pan C, Li T (2018). Abnormally low Bispectral index and severe hypoglycemia during maintenance of and recovery from general anesthesia in diabetic retinopathy surgery: two case reports. BMC Anesthesiology, 18:1-4.

[66] Hwang JJ, Parikh L, Lacadie C, Seo D, Lam W, Hamza M, et al. (2018). Hypoglycemia unawareness in type 1 diabetes suppresses brain responses to hypoglycemia. J Clin Invest, 128:1485-1495.

[67] Jackson DA, Michael T, Abreu AVd, Agrawal R, Bortolato M, Fisher SJ (2018). Prevention of Severe Hypoglycemia-Induced Brain Damage and Cognitive Impairment with Verapamil. Diabetes, 67:2107-2112.

[68] Rehni AK, Shukla V, Perez-Pinzon MA, Dave KR (2018). Acidosis mediates recurrent hypoglycemiainduced increase in ischemic brain injury in treated diabetic rats. Neuropharmacology, 135:192-201.

[69] Faiq MA, Dada R, Saluja D, Dada T (2014). Glaucoma- 
Diabetes of the brain: A radical hypothesis about its nature and pathogenesis. Med Hypotheses, 82:535-546.

[70] Kandimalla R, Thirumala V, Reddy PH (2017). Is Alzheimer's disease a Type 3 Diabetes? A critical appraisal. Biochim Biophys Acta, 1863:1078-1089.

[71] Chouraki V, Preis SR, Yang Q, Beiser A, Li S, Larson $\mathrm{MG}$, et al. (2017). Association of amine biomarkers with incident dementia and Alzheimer's disease in the Framingham Study. Alzheimer's \& Dementia, 13:13271336.

[72] Domínguez RO, Pagano MA, Marschoff ER, González SE, Repetto MG, Serra JA (2014). Alzheimer disease and cognitive impairment associated with diabetes mellitus type 2: associations and a hypothesis. Neurología, 29:567-572.

[73] Pardeshi R, Bolshette N, Gadhave K, Ahire A, Ahmed S, Cassano T, et al. (2017). Insulin signaling: An opportunistic target to minify the risk of Alzheimer's disease. Psychoneuroendocrinology, 83:159-171.

[74] Beheshti I, Mishra S, Sone D, Khanna P, Matsuda H (2020). T1-weighted MRI-driven Brain Age Estimation in Alzheimer's Disease and Parkinson's Disease. Aging Dis, 11:1-11.

[75] Zhang S, Zhi Y, Li F, Huang S, Gao H, Han Z, et al. (2018). Transplantation of in vitro cultured endothelial progenitor cells repairs the blood-brain barrier and improves cognitive function of APP/PS1 transgenic AD mice. J Neurol Sci, 387:6-15.

[76] Baglietto-Vargas D, Shi J, Yaeger DM, Ager R, LaFerla FM (2016). Diabetes and Alzheimer's disease crosstalk. Neurosci Biobehav Rev, 64:272-287.

[77] Santos CaY, Snyder PJ, Wu W-C, Zhang M, Echeverria A, Alber J (2017). Pathophysiologic relationship between Alzheimer's disease, cerebrovascular disease, and cardiovascular risk: A review and synthesis. Alzheimer's \& Dementia: Diagnosis, Assessment \& Disease Monitoring, 7:69-87.

[78] Zhang J, Chen C, Hua S, Liao H, Wang M, Xiong Y, et al. (2017). An updated meta-analysis of cohort studies: Diabetes and risk of Alzheimer's disease. Diabetes Res Clin Pr, 124:41-47.

[79] Degen C, Toro P, Schönknecht P, Sattler C, Schröder J (2016). Diabetes mellitus Type II and cognitive capacity in healthy aging, mild cognitive impairment and Alzheimer's disease. Psychiatry Res:42-46.

[80] Vieira MNN, Lima-Filho RAS, Felice FGD (2018). Connecting Alzheimer's disease to diabetes: Underlying mechanisms and potential therapeutic targets. Neuropharmacology, 136:160-171.

[81] Saraswati AP, Hussaini SMA, Krishna NH, Babu BN, Kamal A (2018). Glycogen synthase kinase-3 and its inhibitors: Potential target for various therapeutic conditions. Eur J Med Chem, 144:843-858.

[82] Zhang Y, Huang N-q, Yan F, Jin H, Zhou S-y, Shi J-s, et al. (2018). Diabetes mellitus and Alzheimer's disease: GSK-3 $\beta$ as a potential link. Behav Brain Res, 339:57-65.

[83] Kubis-Kubiak A, Rorbach-Dolata A, Piwowar A (2019). Crucial players in Alzheimer's disease and diabetes mellitus: Friends or foes? Mechanisms of Ageing and Dev, 181:7-21.
[84] Kinney JW, Bemiller SM, Murtishaw AS, Leisgang AM, Salazar AM, Lamb BT (2018). Inflammation as a central mechanism in Alzheimer's disease. Alzheimer's \& Dementia: Translational Res Clin Interventions, 4:575590.

[85] Felice FGD, Lourenco MV, Ferreira ST (2014). How does brain insulin resistance develop in Alzheimer's disease? Alzheimer's \& Dementia, 10:S26-S32.

[86] Mushtaq G, Khan JA, Kumosani TA, Kamal MA (2015). Alzheimer's disease and type 2 diabetes via chronic inflammatory mechanisms. Saudi J Biol Sci, 22:4-13.

[87] C.Bir S, Y.Chernyshev O, Minagar A (2018). Chapter 27-Roles of Toll-Like Receptors in Pathophysiology of Alzheimer's Disease and Multiple Sclerosis. Neuroinflammation (Second Edition):541-562.

[88] Huang N-Q, Jin H, Zhou S-y, Shi J-s, Jin F (2017). TLR4 is a link between diabetes and Alzheimer's disease. Behav Brain Res, 316:234-244.

[89] Velazquez R, Tran A, Ishimwe E, Denner L, Dave N, Oddo S, et al. (2017). Central insulin dysregulation and energy dyshomeostasis in two mouse models of Alzheimer's disease. Neurobiol Aging, 58:1-13.

[90] Shinohara M, Sato N (2017). Bidirectional interactions between diabetes and Alzheimer's disease. Neurochem Int, 108:296-302.

[91] Han C, Rice MW, Cai D (2016). Neuroinflammatory and autonomic mechanisms in diabetes and hypertension. Am J Physiol Endocrinol Metab, 311:E32-E41.

[92] Muriach Ma, Flores-Bellver M, Romero FJ, Barcia JM (2014). Diabetes and the Brain: Oxidative Stress, Inflammation, and Autophagy. Oxid Med Cell Longev, 2014:1-9.

[93] Chen Y-L, Weng S-F, Yang C-Y, Wang J-J, Tien K-J (2019). Diabetic ketoacidosis further increases risk of Alzheimer's disease in patients with type 2 diabetes. Diabetes Res Clin Pr, 147:55-61.

[94] Tang Y, Li Y-M, Zhang M, Chen Y-Q, Sun Q (2019). ع3/4 genotype of the apolipoprotein $\mathrm{E}$ is associated with higher risk of T Alzheimer's disease in patients with type 2 diabetes mellitus. Gene, 703:65-70.

[95] Valente T, Gella $A$, Fernàndez-Busquets $X$, Unzeta $M$, Durany N (2010). Immunohistochemical analysis of human brain suggests pathological synergism of Alzheimer's disease and diabetes mellitus. Neurobiol Dis, 37:67-76.

[96] Mittal K, Katare DP (2016). Shared links between type 2 diabetes mellitus and Alzheimer's disease: A review. Diabetes \& Metabolic Syndrome: Clin Res Rev, 10S:S144-S149.

[97] Li J, Cesari M, Liu F, Dong B, Vellas B (2017). Effects of Diabetes Mellitus on Cognitive Decline in Patients with Alzheimer Disease: A Systematic Review. Can J Diabetes, 41:114-119.

[98] Clark LR, Berman SE, Rivera-Rivera LA, Hoscheidt SM, Darst BF, Engelman CD, et al. (2017). Macrovascular and microvascular cerebral blood flow in adults at risk for Alzheimer's disease. Alzheimer's \& Dementia: Diagnosis, Assessment \& Disease Monitoring, 7:48-55.

[99] Gej1 M, Gjedde A, Brock B, Møller A, Duinkerken Ev, Haahr HL, et al. (2018). Effects of hypoglycaemia on 
working memory and regional cerebral blood flow in type 1 diabetes: a randomised, crossover trial. Diabetologia, 61:551-561.

[100] Steculorum SM, Solas M, Brüning JC (2014). The paradox of neuronal insulin action and resistance in the development of aging-associated diseases. Alzheimer's \& Dementia, 10:S3-S11.

[101] Assy MH, Awd M, Elshabrawy AM, Gharieb M (2019). Effect of Ramadan fasting on incidence of cerebrovascular stroke in Egyptian patients with Type 2 Diabetes Mellitus. Res Clin Pr, 151:299-304.

[102] Luitse MJA, Biessels GJ, Rutten GEHM, Kappelle LJ (2012). Diabetes, hyperglycaemia, and acute ischaemic stroke. Lancet Neurol, 11:261-271.

[103] Mangin G, Poittevin M, Charriaut-Marlangue C, Giannesini C, Merkoulova-Rainon T, Kubis N (2019). Glatiramer acetate reduces infarct volume in diabetic mice with cerebral ischemia and prevents long-term memory loss. Brain Behav Immun, 80:315-327.

[104] Filipov A, Ebert AD, Neumaier-Probst E, Alonso A (2018). The Burden of Diabetes and the Chance of a Previous Stroke: Thrombolysis for Recurrent Stroke in Diabetics. J Stroke Cerebrovascular Dis, 27:13431349.

[105] Chen R, Ovbiagele B, Feng W (2016). Diabetes and Stroke: Epidemiology, Pathophysiology, Pharmaceuticals and Outcomes. Am J Med Sci, 351:380-386.

[106] Shimizu Y, Harashima A, Munesue S, Oishi M, Hattori T, Hori O, et al. (2020). Neuroprotective Effects of Endogenous Secretory Receptor for Advanced Glycation End-products in Brain Ischemia. Aging Dis, 11:1-12.

[107] Braun KF, Otter W, Sandor SM, Standl E, Schnell O (2012). All-cause in-hospital mortality and comorbidity in diabetic and non-diabetic patients with stroke. Diabetes Res Clinical Pr, 98:164-168.

[108] Alloubania A, Saleh A, Abdelhafiz I (2018). Hypertension and diabetes mellitus as a predictive risk factors for stroke. Diabetes \& Metabolic Syndrome: Clin Res Rev, 12:577-584.

[109] Chen G, Wang X, Robinson TG, Pikkemaat M, Lindley RI, Zhou S, et al. (2018). Comparative effects of low-dose versus standard-dose alteplase in ischemic patients with prior stroke and/or diabetes mellitus: The ENCHANTED trial. J Neurol Sci, 387:1-5.
[110] Akhtar N, Kamran S, Singh R, Malik RA, Deleu D, Bourke PJ, et al. (2019). The Impact of Diabetes on Outcomes After Acute Ischemic Stroke: A Prospective Observational Study. J Stroke Cerebrovascular Dis, 28:619-626.

[111] Pan Y, Chen W, Wang Y (2019). Prediabetes and Outcome of Ischemic Stroke or Transient Ischemic Attack: A Systematic Review and Meta-analysis. J Stroke Cerebrovascular Dis, 28:683-692.

[112] Sun Y, Toh MPHS (2009). Impact of Diabetes Mellitus (DM) on the Health-Care Utilization and Clinical Outcomes of Patients with Stroke in Singapore. Value Health, 12:S101-S105.

[113] Elgebaly MM, Arreguin J, Storke N (2019). Targets, Treatments, and Outcomes Updates in Diabetic Stroke. J Stroke Cerebrovascular Dis, 28:1413-1420.

[114] Jun-Min C, Xiang-Jian Z, Xiao-Xia L, De-Gang S, Xiao-Yun C, Qian S (2018). Pericytes and Central Nervous System Diseases. Chin J Stroke, 13:90-95.

[115] Zhong W, Yuan Y, Gu X, Kim SI-y, Chin R, Loye M, et al. (2020). Neuropsychological Deficits Chronically Developed after Focal Ischemic Stroke and Beneficial Effects of Pharmacological Hypothermia in the Mouse. Aging Dis, 11:1-16.

[116] Yan W, Zhao X, Chen H, Zhong D, Jin J, Qin Q, et al. (2016). $\beta$-Dystroglycan cleavage by matrix metalloproteinase-2/-9 disturbs aquaporin-4 polarization and influences brain edema in acute cerebral ischemia. Neuroscience, 326:141-157.

[117] Villalba H, Shah K, Albekairi TH, Sifat AE, Vaidya B, Abbruscato TJ (2018). Potential role of myo-inositol to improve ischemic stroke outcome in diabetic mouse. Brain Res, 1699:166-176.

[118] Fuentes B (2018). Antidiabetic drugs for stroke prevention in patients with type-2 diabetes. The neurologist's point of view. Medicina Clinica, 150:275-281.

[119] Rehni AK, Liu A, Perez-Pinzon MA, Dave KR (2017). Diabetic aggravation of stroke and animal models. Exp Neurol, 292:63-79.

[120] Boyko M, Zvenigorodsky V, Grinshpun J, Shiyntum HN, Melamed I, Kutz R, et al. (2019). Establishment of novel technical methods for evaluating brain edema and lesion volume in stroked rats: A standardization of measurement procedures. Brain Res, 1718:12-21. 